\title{
MicroRNAs in Psychological Stress Reactions and Their Use as Stress-Associated Biomarkers, Especially in Human Saliva
}

\author{
Conrad Wiegand $^{a} \quad$ Andreas Savelsbergh $^{b}$ Peter Heusser ${ }^{a}$ \\ anstitute of Integrative Medicine, University of Witten/Herdecke, Herdecke, \\ ${ }^{b}$ Chair for Biochemistry and Molecular Medicine, Division of Functional Genomics, \\ Center for Biomedical Education and Research (ZBAF), University of Witten/Herdecke, \\ Witten, Germany
}

\begin{abstract}
What Is It about?
Chronic and acute psychological stress plays a major role in many diseases. MicroRNAs (miRNAs) are largely integrated in the regulation of these stress reactions. They are present in all body fluids, making them possible biomarkers for the determination of stress reactions and, possibly, the related coping mechanisms. Salivary miRNAs can be obtained least invasively and might provide a suitable source for the assessment of stress-related conditions.
\end{abstract}

\section{Keywords}

MicroRNA · Salivary microRNA · Psychological stress · Social stress · Mental stress ·

Epigenetics · Epigenetic biomarker

\begin{abstract}
MicroRNAs (miRNAs) play a central role in the regulation of many cellular processes including physiological and psychological stress reaction pathways. Psychological stress is an important factor for the genesis and maintenance of many diseases. Several miRNAs have already been described to be involved in its regulation. The presence of miRNAs in all body fluids implies a widespread role in communication throughout the whole organism and together with their stability makes them formidable candidates as biomarkers. Alterations of stress-associated miRNA expression levels have been found in the brain and whole blood of humans and animals. In this paper, we review the participation of miRNAs in stress-reactive processes as well as their usability as salivary biomarkers of such processes. In conclusion, we suggest that salivary miRNAs may be useful as noninvasive biomarkers to assess epigenetic regulation processes of chronic or acute psychological stress reactions.

(C) 2017 The Author(s)

Published by S. Karger AG, Basel
\end{abstract}

Andreas Savelsbergh, PD Dr.

Center for Biomedical Education and Research (ZBAF)

University of Witten/Herdecke

Stockumer Strasse 10, DE-58453 Witten (Germany)

E-Mail andreas.savelsbergh @ uni-wh.de 


\section{Introduction}

Stress plays an important role in daily life and can be caused by a variety of stressors that potentially threaten an individual's homeostasis, well-being, overall health, or survival [1]. Stress triggers a coordinated systemic physiological stress response, including inflammatory, cellular, and metabolic processes as well as their epigenetic regulation. Due to the duration of the stressors influence, the stress response can be divided into acute and chronic stress reactions, and it occurs in several forms [1]. The perception and experience of psychological stress is subjective and individual. There are large interindividual differences and variations of perceived stress and stress reactions. The same applies to the individual coping mechanisms of an organism, which depend on several individual factors [2].

As long as the coping processes of the organism suffice to deal with the stressor, particularly acute psychological stress can induce positive, beneficial, and adaptive effects $[1,2]$. Such beneficial stress, not exceeding the coping capacity of an organism is called "eustress" $[2,3]$.

If the stressor's influence exceeds the individual coping capacity due to intensity or duration, psychological stress can induce harmful physiological effects. Thus, stress is an important cofactor for the genesis and maintenance of acute and chronic diseases such as diabetes mellitus, asthma bronchiale, arteriosclerosis, and ulcerative colitis, but also neuroses, depression, or schizophrenia [2,4].

Stress also has an effect on gene expression and posttranscriptional mechanisms via epigenetic regulation. This has already been described for asthma, arthritis, and other inflammatory diseases [4].

miRNAs are small, noncoding RNAs, 22-23 nucleotides in length, which play an important regulatory role in gene expression. Targeting messenger RNAs (mRNAs), they induce their cleavage, degradation, or translational repression $[5,6]$. The transcription of DNA coding for miRNAs occurs in a similar way as in protein-coding genes [6,7]. The mature miRNA directly interacts with a member of the Argonaute protein family, forming the RNA-induced silencing complex (RISC) [5]. As a component of RISC, miRNAs direct the posttranscriptional repression $[6,8]$.

Until now, more than 950 miRNAs in humans have been registered in the miRBase release (http://www.mirbase.org) [9]. miRNAs were detected to have up to hundreds of targets each [10]. Due to these regulatory functions in gene expression, miRNAs play a central role in several cellular processes, including cell growth, differentiation, proliferation, and apoptosis [11].

miRNAs exert important functions in the epigenetic regulation of inflammatory and autoimmune processes of chronic diseases such as asthma or arthritis [12], whose genesis is linked to stress [4]. Additionally, miRNAs take part in the regulation of gene expression under mental stress exposure [13-16].

As stress is an important cofactor in the genesis and maintenance of a variety of acute and chronic diseases such as cardiovascular disease, respiratory infections, and chronic liver disease as well as several types of psychiatric disorders $[17,18]$, stress is of high epidemiological and health-economic relevance [19].

Playing an important role in the epigenetic regulation of stress reactions [16], miRNAs might not only be used to elucidate these reactions as such, but also as biomarkers in the assessment of these reactions as well as in the diagnosis, prevention, and treatment evaluation of stress-associated diseases. Indeed, miRNAs can be identified in several body fluids, including saliva $[13,20]$. For reasons of ethics and practicality, the optimal method for obtaining miRNAs for biomarker purposes would be the least invasive one, namely from saliva. However, to our knowledge, stress-associated salivary miRNAs have not been used as biomarkers for stress-associated psycho-physiological reactions, so far. 
Fig. 1. Overview of the results of the literature search in PubMed.

2017 The Author(s). Published by S. Karger AG, Basel www.karger.com/bmh

Wiegand et al.: MicroRNAs in Psychological Stress Reactions and Their Use as Stress-Associated Biomarkers, Especially in Human Saliva

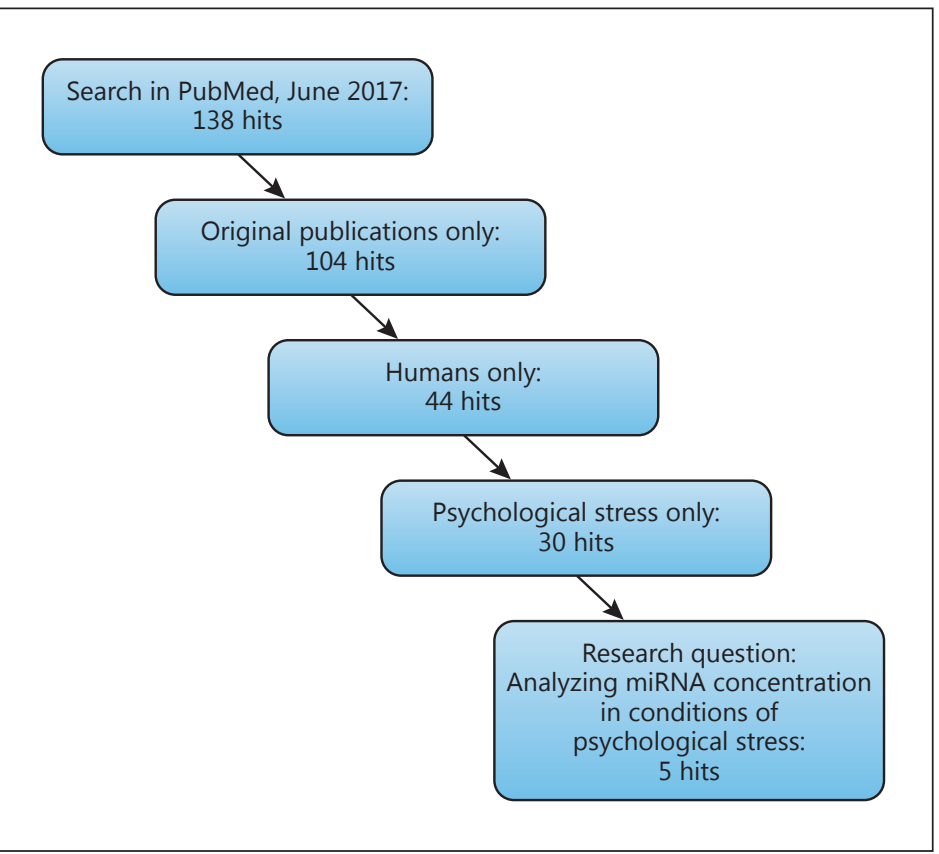

The aims of this paper are (1) to review present knowledge on the involvement of miRNAs in acute and chronic psychological stress reactions in humans; (2) to give a brief overview over the physiological, cellular, and molecular reactions to psychological stress; (3) to review current knowledge on the functional roles of miRNAs in the context of these stress reactions and stress-associated processes; (4) to review the use of miRNAs as biomarkers in general; and (5) to review available evidence indicating whether miRNAs obtained from saliva might be useful as biomarkers for psychological stress reactions and stress-induced conditions.

\section{Methods}

The literature search for our review was divided into 2 parts: a systematic search on the involvement and role of miRNAs in psychological stress reactions and a narrative part, explaining the background of miRNAs and providing explanation models of their involvement and their role in such reactions.

For the first part, we searched in PubMed using the search terms: "(miRNA OR microRNA) AND (psychological OR mental OR social) AND stress."

We integrated papers which were of experimental or clinical character (reviews were excluded), concerned only human subjects (studies on animals or plants were excluded), used psychological stressors (either acute or chronic), and analyzed alterations in miRNA levels as a research question (Fig. 1).

Based on this, we continued our literature search, analyzing the background for miRNA involvement in psychological stress reactions, as the second part of our review. We extended our search looking for publications concerning (a) the involvement of miRNA in physiological stress reactions, (b) their involvement in stress-associated diseases, and (c) a possible use of miRNAs in human body fluids as biomarkers for psychological stress reactions, - especially in saliva as a noninvasive source. This was made by thematically continuing our search based on our previous findings, and not systematically by using fixed search terms. By this, we 
Table 1. Important references

\begin{tabular}{|c|c|}
\hline First author [Ref.], year & Key topic \\
\hline Beech [22], 2014 & $\begin{array}{l}\text { miRNA expression in human whole blood in an experimental setting of acute } \\
\text { psychological stress }\end{array}$ \\
\hline Vaisvaser [21], 2016 & $\begin{array}{l}\text { miRNA expression in human whole blood in an experimental setting of acute } \\
\text { psychological stress }\end{array}$ \\
\hline Katsuura [13], 2012 & $\begin{array}{l}\text { miRNA expression in human whole blood in conditions of chronic } \\
\text { psychological stress }\end{array}$ \\
\hline Honda [23], 2013 & $\begin{array}{l}\text { miRNA expression in human whole blood in conditions of chronic } \\
\text { psychological stress }\end{array}$ \\
\hline Gidron [24], 2010 & $\begin{array}{l}\text { miRNA expression in human whole blood in conditions of chronic } \\
\text { psychological stress }\end{array}$ \\
\hline Bartel [6], 2009 & Background and introduction to the field of miRNAs \\
\hline Leung [16], 2010 & Involvement of miRNAs in several stress-responsive processes \\
\hline Michael [20], 2010 & miRNAs in human saliva as biomarker for several processes \\
\hline Tsai [11], 2010 & Involvement of miRNAs in several common diseases \\
\hline Dirven [55], 2017 & $\begin{array}{l}\text { Regulatory involvement of miRNAs and other epigenetic factors in the } \\
\text { neuroendocrine stress response }\end{array}$ \\
\hline
\end{tabular}

intended to describe the background and possible explanation models for the involvement and the role of miRNAs in psychological stress reactions, as well as their possible use as biomarkers.

For this part, no specific inclusion or exclusion criteria were used. Every type of papers including reviews, experimental or clinical studies, either on humans or animals, was included. The selection of papers was not made systematically, but in a causal context. Our literature search includes publications which were released up until June 2017.

\section{Results}

Involvement of miRNAs in Acute and Chronic Psychological Stress Reactions in Humans

Screening the current literature for the involvement and the role of miRNAs in psychological stress response systematically, as indicated in the methods section, in the PubMed library up until June 2017, 138 hits were found. Regarding our inclusion and exclusion criteria (see "Methods"), 5 papers remained for further analysis (see Table 1).

All of these studies investigated alterations in miRNAs in human whole blood, either in acute or chronic psychological or in mental or social stress conditions, respectively. Studies investigating miRNAs in other body fluids or tissue of humans in such conditions were not found.

Two of these studies used experimental stress elicitation tasks - analyzing acute, not chronic stress reactions [21,22]. Three of these studies used stressors of everyday life, such as academic exams or tests as events to be expected and therefore functioning as chronic psychological stressors [13, 23, 24].

Beech et al. [22] investigated miRNA expression in an acute stress response in humans. They used an imaginary script development procedure to induce a condition of acute psycho- 
logical stress. They found an increase in miR-10a and miR-21 in whole blood samples of the participants, collected $1 \mathrm{~h}$ after the stress induction. Furthermore, the authors suggested changes in specific gene expressions, which might be related to the upregulation of these miRNAs. As a conclusion of these results, it can be suggested that miR-10a and miR-21 expression in whole blood is regulated by acute psychological stress in this context [22].

In 2016, Vaisvaser et al. [21] investigated miRNA expression in peripheral blood, responding to an acute psychological stress elicitation task. They found an increase in the concentration levels of miR-29c in peripheral blood. Changes in the miRNA concentration levels correlated with the subjective experience of perceived stress (measured by a 9 point Likert scale) and the activation of the ventromedial prefrontal cortex as a key area of stress regulation (measured in parallel by functional magnetic resonance imaging). They assumed that stress-induced changes in miRNA expression may occur very quickly - minutes after induction [21].

In 2012 [13] and 2013 [23], a group of scientists from the Department of Stress Science at the University of Tokushima, Japan, investigated alterations in miRNAs in the whole blood in conditions of chronic psychological stress. Katsuura et al. [13] examined healthy fourthgrade medical students, taking a nationally administered examination for academic promotion. Changes in 11 miRNAs were detected in whole blood (miR-15a, -16, - 19a, -19b, -26b, -30b, $-106 \mathrm{~b},-126,-142-3 \mathrm{p},-144$, and $-144^{*}$ ), comparing 4 different time points (7 weeks before, 1 day before, immediately after and 1 week after the examination). Six of these miRNAs had already been reported to be associated with psychological stress response or psychiatric disorders (miR-16, -19a, -19b, -26b, -144, and -144*) [25]. Most of these miRNAs increased and peaked immediately after the examination and returned to baseline 1 week after it. In the cases of miR-16, -144 , and $-144^{*}$, these changes were statistically significant. Additionally, correlations with other stress-responsive parameters were analyzed by measurements of IL-1 $\beta$, IL- 6 , TNF- $\alpha$, and IFN- $\chi$ in the serum, as well as salivary cortisol and the performance of a state anxiety score as a psychological stress assessment. Some significant correlations between certain miRNAs and cytokines like TNF- $\alpha$ and IFN- $\chi$ were found, showing also impermanent correlations with cortisol levels, but no significant correlation with the state anxiety score.

Interpreting these results, Katsuura et al. [13] mentioned: "miR-144* and miR-16 might be one of the negative regulators of inflammatory cytokine response after exposure to naturalistic stressors."

Based on these investigations, Honda et al. [23] examined 25 male, healthy medical students preparing for the National Examination for Medical Practitioners, a 3-day test which has to be passed to become a clinical physician. Saliva and peripheral blood were collected at 3 time points; 2 months before the exam, 2 days before and 1 month after the exam, measuring salivary cortisol levels and miRNA expression levels in whole blood. At the same time points, all subjects answered the state version of Spielbergers' state-trait anxiety inventory (STAI) to assess their perceived psychological stress level $[26,27]$. The results of STAI confirmed that this study set-up was sufficient to induce chronic psychological stress and to use miRNAs as biomarkers for it. After statistical analysis 7 miRNAs (miR-16, -20b, -26b, -29a, -126, -144, and $-144^{*}$ ) were identified to show significant responses to the examination stress. Correlations between the miRNAs and the other parameters were analyzed, showing significant correlations between certain miRNAs and the STAI scores, but no significant correlation with the salivary cortisol levels. Interpreting these results, the authors suggest that changes in the miRNA concentration levels, focusing on miR-16, might be related to anxiety rather than cortisol responses.

Interestingly, Honda et al. [23] found that the stress-dependent concentration levels of miRNA in the peripheral blood of their healthy subjects were smaller than in Alzheimer 
disease [28], depression [29], and autism [30], which had been investigated in previous studies [23].

Two years before, in 2010, Gidron et al. [24] had compared the concentration-levels of 2 miRNAs, let7-b and miR-21, in periods of low stress and high stress; 37 medical students were tested. Quantitative PCR measurements were used for the miRNAs. For the low-stress period at baseline, blood samples were taken directly after the end of a study break. For the highstress period, blood samples were taken directly after an academic exam. The Perceived Stress Questionnaire [31] was used to assess the levels of perceived stress at both time points. Perceived stress levels in the high-stress period were significantly higher. Furthermore, the health behavior of the participants was assessed at baseline, using a specific questionnaire [32]. Comparing the expression levels of let-7b and miR-21 in the whole blood of the participants at both time points, a significant decrease in both was found from low-stress/baseline to the high-stress period. While this decrease was found for let-7b in all participants, interestingly such a decrease was found for miR-21 only in the participants with inadequate health behavior, but not with adequate health behavior. Interpreting these results, the authors suggested miR-21 to be possibly useful as a biomarker in human whole blood for psychological stress. As limitations of their study, the authors acknowledged that it did not use an experimental design and had no control group [24].

Summarizing, it can be said that some investigations on miRNA involvement in psychological stress reactions in humans were already done. All of the studies described stress-associated changes in the concentration levels of specific miRNAs in human whole blood and therefore provide the suggestion of possibly useful biomarkers in human whole blood. Interestingly, no other source of human body fluids was used in these studies. The main limitation of all of these studies is that no control groups were used. While some of the studies were of experimental character, others were not.

Summing up these findings, there remains only invalid evidence for the use of specific miRNAs as biomarkers in psychological stress reactions. Nevertheless, a handful of miRNAs has been found to be altered in response to psychological stress. As only first steps of investigation have been made in this field of research, it is important to scrutinize the theoretical background and explanation models for the involvement of miRNAs in psychological stress reactions and stress-associated processes.

Physiological, Cellular, and Molecular Reactions to Psychological Stress

To provide a comprehensive understanding of the functional roles of miRNAs in psychological stress reactions, we first provide a brief overview of the physiological, cellular, and molecular reactions to psychological stress.

Facing a psychological stressor, the stress response usually starts with impulses from high cortical centers of the brain that are relayed through the limbic system to the hypothalamus [33]. The acute response to the stressor is then induced in the limbic system where the subjective evaluation of this stimulus takes place in the context of the individual coping capacity [33] and the respective personality traits [34]. The physiological response to such a stressor is structured in 2 successive processes.

First, the "alarm" response is induced, resulting in the activation of the autonomic nervous system (ANS), which leads to an increased release of epinephrine and norepinephrine from the adrenal medulla [1] as well as serotonin and adrenaline in the central and peripheral nervous system [33]. This reaction is also called the sympathetic-adrenal-medullary (SAM) axis [35] and aims at the capacity to "fight-fright-or-flight." It concentrates the resources of the organism on the necessary organs to fight or flight, increasing the blood flow to the heart and skeletal muscle, the metabolic rate, the blood pressure, and the respiration [1]. 
With a short delay, the hypothalamic-pituitary-adrenal (HPA) axis is activated as the second stage, aiming to provide the energy resources for a longer period of time than the SAM is able to [1]. The intensity of the reaction and the manner of inducing stress reactions is of a very individual nature. The HPA axis affects the organism mainly in a slow and genomic manner through a transcriptional regulation of glucocorticoid-responsive genes, determining the magnitude and specificity of an individual's behavioral, neuronal and hormonal response to stress [1].

Through these reactions, psychological stress influences a host of processes in the human organism such as the metabolic or immune systems [2,34]. In this respect, acute stress often leads to positive and beneficial effects such as a boost of the immune system, while persisting chronic stress is associated with pathological processes and disturbance of immune functions, mainly by the activation of cortisol; and it possibly leads to a variety of different diseases [36]. Additionally, the exposure to chronic stress affects neuronal plasticity. Profound effects on the structural and functional integrity of the brain have already been described and reviewed in recent literature [1].

The individual subjective stress perception depends on personality traits [34] and personal coping mechanisms [2]. The psychophysiological stress response can be assessed by stress-reactive molecules such as salivary cortisol as a measure of the HPA axis [37] or salivary $\alpha$-amylase as a measure of the SAM axis [38]. Individual differences in the extent of the stress response can be seen in standardized psycho-social stress tests by analyzing these parameters [34, 39].

As to the cellular level, an increase in circulating cytokines has been found under psychological stress exposure due to the activation of the HPA axis and cortisol, inducing inflammatory reactions and disturbance of the cellular homeostasis [40] and causing cellular stress. Cellular stress can be defined as a deviation from the status quo [16], or "a reaction to the threat of macromolecular damage" of membrane lipids, proteins, and/or DNA caused by external or internal stimuli such as oxidative stress [41].

Oxidative stress occurs when there is an imbalance between the concentration of reactive oxygen species (ROS) and the capability to detoxify them. ROS mainly arise from activation of the respiratory chain in the mitochondria. As a side product of cellular metabolism, they are produced also in nonstressed cells, but their abundance increases through increased metabolic activation [42], e.g. due to activation of the HPA axis. ROS affect and damage several different cellular biomolecules and cell organelles, disturbing their physiological processes and functions.

This molecular damage induces different specific pathways, which can be summarized as the "cellular stress response" to cope with the stressor: (1) the oxidative stress response, (2) the heat stress response, (3) the unfolded protein response (UPR), and (4) the DNA damage response [42]. While the oxidative stress response mainly describes the enzymatic response to the accumulation of ROS, the other pathways are induced by damaged proteins (UPR and heat shock response) or damaged DNA (DNA damage response). Each stress response aims to recreate cellular homeostasis by the induction of respective individual repair mechanisms, or - if the coping capacity is exceeded - to induce apoptosis, preventing the organism from high systemic damage.

Additionally, there is evidence suggesting that chronic psychosocial stress may also lead to DNA damage. This includes damage of mitochondria and mitochondrial DNA in the metabolic wake of stress-induced glucocorticoid dysregulation, but also telomere shortening due to stress-related oxidative damage, processes which can induce systemic inflammation, alter gene expression, accelerate cell ageing, and possibly also the development of cancer [43-46]. 


\section{Functional Roles of miRNAs in Stress Reactions and Stress-Associated Processes}

As already mentioned, stress-responsive pathways are already well understood or in the process of being further elucidated. But as the regulatory signaling mechanisms of the specific pathways are concerned, larger knowledge gaps remain. Recent studies have accumulated evidence for miRNAs to play a key role in the molecular regulation of these pathways [16]. This happens by modulating the amount of miRNAs, the amount of mRNA targets, or the activity and mode of action of RISCs. Thus, miRNAs are assumed to be important regulators of the homeostasis of cells when exposed to environmental stress factors [16]. In this section, we review the functional roles of miRNAs in (a) cellular and physiological stress response pathways, (b) in psychological stress-associated processes, and (c) in stress-associated neurological and psychiatric disorders.

miRNAs in Cellular and Physiological Stress Response Pathways

miRNAs are functionally involved in the up- or downregulation of a variety of different cellular stress-responsive pathways. The oxidative stress response is a pathway which provides and activates several antioxidant proteins and enzymes such as glutathione or superoxide dismutase [42], both of which are directly regulated by different miRNAs to detoxify oxidative stress metabolites $[47,48]$. Both, the heat stress response and the UPR are pathways meant to cope with the accumulation of misfolded proteins, mostly in the endoplasmic reticulum [40]. Different miRNAs were found to be involved in their signaling cascades $[49,50]$. For example, miR-211 shows time-dependent changes in its concentration levels, providing an antiapoptotic signal by repressing proapoptotic factors in the first hours after stress induction, changing to a proapoptotic signal after exceeding these first hours of the UPR [51]. The DNA damage response is a pathway which provides cell cycle arrest or even apoptosis through p53 induction, when also the DNA is affected by oxidative stress [52]. It is well established that the miR-34 family is involved as a key molecule in the p53 system [15].

This array of miRNAs involved in the cellular stress response pathways is certainly far from complete, but it reasonably suggests the functional involvement of miRNAs in the regulation of cellular response processes to psychological stress.

In regard of organismic stress reactions on a supracellular, i.e. physiological, level mainly 2 types of response to stress in general - either to an intrinsic or extrinsic stressor - are commonly distinguished: (a) the "alarm response," mainly consisting in the activation of ANS and SAM, and (b) the activation of the HPA axis, mediated by cortisol as the "delayed response." While numerous of the retrieved studies clearly suggest a regulatory role of miRNAs in the "delayed response," no conclusive relation between miRNA expressions and the regulation of ANS and SAM activation could be found. There are just a few references indicating that certain miRNAs are involved in the regulation of serotonin effects as one of the mechanisms of the ANS response to psychological stress, in that they influence the cellular serotonin uptake by affecting the serotonin transporter [53].

Focusing on the regulation of the HPA axis by cortisol, more references were found on the involvement of miRNAs in its regulation (see below). For example, miRNAs exert a regulatory function in the cortisol production as the most important signaling component in this response. miR-24 has been found to regulate the production of aldosterone and cortisol in the adrenal cortex. By targeting 2 of the key enzymes of their production, CYP11B1 and CYP11B2, miR-24 significantly decreases the production of aldosterone and cortisol in adrenocortical cells [54]. Therefore, it might be assumed that stress-related changes in miRNA-24 expression levels might constitute an adapting mechanism of the organismic response to psychological stress by influencing the subjective stress resilience, either in a positive or negative manner. Aside from miRNAs, other epigenetic mechanisms such as DNA methylation and histone modification were found to regulate the neuroendocrine stress response, mainly by regu- 
lating glucocorticoid or mineralocorticoid receptors. Dirven et al. [55] provided a detailed analysis of this epigenetic regulation of HPA axis activation in their review.

The elucidation of these miRNA functions gives an impression of their extensive participation in the stress-related molecular and physiological processes of the organism. Due to their effects as posttranscriptional repressors of mRNA, they are candidates for a host of potential regulatory roles in many pathways and signaling processes such as the repression or expression of key proteins necessary for the physiological adaption of the organism to changing environmental conditions.

For example, several miRNA-knockout studies demonstrate that miRNAs mainly function as regulators of adaption or coping with changing extrinsic or intrinsic stress conditions. Apparently, the functions of miRNAs identified on the cellular level have consequences for the regulation of physiological reactions, morphological structures or on more complex supracellular levels. Leung and Sharp [16] reviewed such functions of miRNAs in 2010, surveying pertinent animal experiments. In several of the reviewed studies, the investigated miRNA mutants or miRNA-knockout animals were unable to cope with stress due to changing environmental conditions, even though they might have appeared normal under stable conditions.

Changes in miRNA Concentration Levels in Psychological Stress

As initially mentioned, psychological stress leads to stress reactions on supracellular physiological as well as cellular and molecular levels. As shown above, miRNAs appear to be functionally involved and integrated in these processes. Therefore, a change in the concentration of certain miRNAs responding to psychological stress can be expected. As the following study results demonstrate, this can indeed be observed in analyses of changing miRNA concentrations in several different body fluids or tissues of the organism - regardless their specific function - responding to psychological stress in a clinical or laboratory study set-up. Hence, it is understandable that in such instances the assessment of miRNAs may easily be motivated by their potential use as biomarkers, apart from their specific cellular or physiological functions.

Animal experiments in rats and mice were the first to lead to the notion that the alteration of miRNA expression levels is a functional response to psychological stress. In mice exposed to acute restraint stress for about $2 \mathrm{~h}$, a significant increase of 2 miRNAs (miR-26b and let-7a) could be observed in the brain $24 \mathrm{~h}$ afterwards, declining almost to the baseline level 5 days afterwards. These results suggest that acute psychological stress effects miRNA expression quickly but not sustainably [56]. In a comparable study in rats, it was demonstrated that miRNA expression depends on the type of stressor (acute or chronic) and the specific region in the brain (amygdala or hippocampus). In brief, the concentration of miRNA triggered by chronic stress was higher than by acute stress. Furthermore, 2 miRNAs (miR-134 and miR-183) were significantly increased by acute stress [14]. Interestingly, these 2 miRNAs share a number of putative targets [57], such as Zinc finger protein multitype 2 (ZFPM2) which is associated with cardiogenesis and cardiovascular malformations in mammals (www. genecards.org) as well as other pathologies [14].

In another experiment with mice exposed to restraint stress, the mineralocorticoid receptor (MR) was identified as one of the targets of both miR-135a and miR-124 [58], which negatively affect its expression. Exposed to acute restraint stress, the increased MR level in the mice amygdala negatively correlated with the miR-135a and mir-124 expression level [58]. Thus, miR-135a and miR-124 are suggested to play a role in the regulation of MR responding to acute psychological stress.

Moreover, specific miRNAs were identified in rat myocardial tissue to be altered in a psychological restraint stress model. Besides the fact that miRNA expression is tissue specific, 
a difference between acute and chronic stress was found in the expression patterns of miRNAs [59].

Additionally, altered expression levels of various miRNAs have been found in the serum and the amygdala in a model of posttraumatic stress disorder (PTSD) in rats. Five circulating miRNAs in peripheral blood were identified as potential biomarkers of PTSD [60].

As in animals, in humans the hippocampal region is an important neurological mediator of psychological stress reactions. By its integration in the regulation of the HPA axis, it is responsible for the regulation of glucocorticoid secretion. As a tissue densely populated with glucocorticoid receptors, it is highly sensitive to stress and glucocorticoid levels. In a mouse model it was demonstrated that stress and increased glucocorticoid levels have an inhibiting effect on neurogenesis in the hippocampal region [61]. Recent studies demonstrate such an effect of stress and of corresponding glucocorticoid levels on adult hippocampal neurogenesis also in human cell lines [62]. Although the detailed regulation of neurogenesis is not yet completely understood, growing evidence demonstrates that miRNAs are involved in neurogenesis and neuronal plasticity [17]. This leads to the assumption that stress-related miRNAs are functionally involved in the regulation of neurogenesis in the hippocampal region and that they are additionally integrated in the regulation of the HPA axis and the glucocorticoid secretion in stress reactions. Another possible hint that miRNAs may participate in the neuronal stress response in the brain is given by a postmortem brain examination of depressed human suicide subjects. In this examination, a significant alteration (downregulation) and reorganization of miRNA expression in the prefrontal cortex was found [63].

miRNAs in Stress-Associated Neurological and Psychiatric Disorders

Aside from standardized study set-ups investigating changes in miRNA expression responding to psychological stress, such changes can also be observed in several neurological and psychiatric diseases whose genesis and maintenance is at least linked to psychological stress $[17,18]$.

Alterations in several circulating miRNA expression levels have been found in psychiatric disorders such as major depressive disorder [64], schizophrenia [65], autism spectrum disorder [66], bipolar disorder [67], Alzheimer disease [68], and mild cognitive impairment [69]. All of these studies describe a possible use of miRNAs as biomarker for the respective disease, mainly in peripheral blood. Additionally, an association of miRNAs with several types of anxiety disorders has been reported [18]. Interestingly, a reduced global miRNA expression was found in the prefrontal cortex of depressed suicide completers. Furthermore, the authors reported miR-185 to regulate the expression of a specific kinase (TrkB-T1), which has been associated with suicidal behavior [70]. Thus, they demonstrated not only a correlation between the quantitative global expression of these miRNAs and suicide, but also suggest a causal connection between specific miRNA function and suicidal behavior. As mentioned before, miRNA expression in the brain is related to psychological stress. In turn, miRNAs were suggested to contribute to the genesis of several of mental disorders through their epigenetic modulation of gene expression implicated in these disorders [71]. Thus, miRNAs constitute an important factor in the causal connection of psychological stress and mental diseases by changed gene expression. It is important to realize that some of these psychiatric disorders including major depressive disorder and anxiety disorders are associated with acute or chronic stress $[17,18]$.

miR-21 serves as a good example for the connection of miRNAs integrated in oxidative stress-related processes and the genesis of psychiatric disorders. It has a direct inhibiting influence on the superoxide dismutase activation as a key enzyme in oxidative stress response. Therefore, it contributes to the persistence of oxidative stress and hence to the genesis of various diseases, such as Alzheimer diseases and also cancer, diabetes, and cardiac disease [47]. 
In summary, this overview demonstrates the involvement of several miRNAs in stressassociated processes. Even though their specific functional roles often remain unclear, stress-associated changes in the miRNA concentration have already been detected in specific studies.

The Use of miRNAs as Biomarkers in General

Even though the majority of miRNAs are found intracellularly, they do not only play a role in regulating intracellular gene expression. Several studies demonstrate a significant number of miRNAs to be present outside of cells; in several body fluids such as whole blood [13], serum [60], plasma [72], saliva [20], and other body fluids - generally in specific secretory patterns [73]. miRNAs are packed in both microvesicles and exosomes, surrounded and protected by a double lipoprotein membrane [74]. Therefore, they appear to be stable even in changing environmental conditions such as considerable changes in temperature $\left(4-37^{\circ} \mathrm{C}\right)$ [72]. This is valid for various sources in humans, such as in peripheral blood [75], saliva [20], and other body fluids [76].

Due to their stable presence, the alteration in their concentration and their ability to respond to changes in extrinsic or intrinsic conditions in certain patterns, miRNAs appear to be very suitable as biological markers for miRNA-associated pathologies or cellular response processes. In 2010, Tsai and Yu [11] reviewed specific response patterns in miRNA expression in several diseases such as cancer, cardiac or neurodegenerative diseases, and autoimmune/ inflammatory or infectious diseases. The potential use of miRNAs as biomarkers in psychiatric conditions has already been mentioned above.

Indeed, in several recent studies, miRNAs have been described as useful biological markers for several conditions or diseases, mostly miRNAs assessed from peripheral blood. This applies to the field of cancer (gastric and other cancers) [77], to the prognosis and progression of chronic lymphatic leukemia, to metabolic diseases, e.g. the detection of prediabetes, diabetes mellitus, and the assessment of the therapeutic response [78], but also to autoimmune diseases [79].

Salivary miRNAs as Potential Biomarkers for Stress-Induced Reactions and Conditions

A possible source to obtain miRNAs as biomarkers is the extraction from saliva. The first description that miRNAs can be extracted from salivary exosomes in quantities adequate for both quantitative PCR and microarray hybridization was published by Michael et al. [20] in 2010. In 2011, Patel et al. [80] described that miRNAs can be isolated from saliva in such a high resolution that specific changes in their levels can become measurable accurately in various diseases [80]. Compared to the assessment in peripheral blood, salivary miRNA has the advantage that it is a noninvasive method for obtaining miRNA, which might be comparably more feasible and ethically more acceptable for biomarker assessment and monitoring.

Up until now, the potential of salivary miRNAs has mainly been described to be possibly useful for the detection and assessment of cancer, especially for oral cancer (squamous cell carcinoma) [81] esophageal cancer [82], and even pancreatic cancer [83], as well as in sputum as a mix of saliva and tracheal secretion also for non-small cell lung cancer [84]. Aside from cancer diagnostics, specific expression patterns of salivary miRNAs can also be used for the detection of autoimmune diseases. Salivary miRNAs from the parotid and submandibular gland have been described to be useful for the detection of Sjögren syndrome $[85,20]$. 


\section{Summary and Conclusion}

In the first part of our review, we systematically investigated the involvement of human miRNAs in psychological stress reactions. Even though evidence remains far from clear, we found 5 papers reporting measurable stress-associated changes in miRNA concentration in human whole blood. All of them suggest the prospective use of miRNAs as epigenetic biomarkers for such reactions.

In the next sections, we gave a short comprehensive description of physiological, cellular and molecular reactions to psychological stress and reviewed the functional involvement of miRNAs in these reactions. Psychological stress leads to oxidative stress by the induction of the SAM- and later the HPA axis. In turn, oxidative stress causes protein damage, inducing specific cellular stress response pathways to cope with it. Our review revealed that several miRNAs are functionally involved in many different stages of these physiological reactions and cellular stress reaction pathways. In some of them, they appear to play important regulatory roles. Furthermore, alterations of miRNA concentration have been detected in several stress-associated pathological conditions and diseases, e.g. in a whole set of psychiatric diseases.

In general, there is a growing use of such condition-dependent concentration changes for the purpose of applying miRNAs as epigenetic biomarkers. This is true for autoimmune, inflammatory, and metabolic processes as well as for various pathologies and diseases. As alterations of miRNA concentrations have already been found in association with conditions of psychological stress, miRNAs might also be useful as biomarkers for psychological stress reactions. Up until now, stress-associated changes in miRNA concentration have only been described for human whole blood. Due to the use of salivary miRNAs as biomarkers in other fields of research and pathological conditions, we suggest that they might also be useful as biomarkers for psychological stress reactions. As a noninvasive source, the collection of saliva samples would not add additional stress to the subject and might therefore be a very suitable source for biomarkers of psychological stress and its preventive or therapeutic monitoring. To verify this assumption, exploratory and experimental research is needed. We strongly encourage exploring this route.

\section{Limitations of the Study}

We started our investigation with a systematic research in the PubMed library for the involvement of miRNAs in psychological stress reactions. Due to the small number of results, the picture remains incomplete. Therefore, we extended our research, trying to find indications of such an involvement of miRNAs. We provide a detailed analysis of psychological stress reactions in general and involvement of miRNAs in other (stress-associated) processes that are already described in the literature. Nevertheless, this second part remains a narrative review, trying to demonstrate explanation models for our research question. For this part, we did not provide a systematic research, but causal analysis of important components for this assumption. Therefore, statements and the collection of results in this second part are limited to our subjective point of view.

\section{Disclosure Statement}

The authors declare no conflicts of interest. 


\section{References}

1 Lucassen PJ, Pruessner J, Sousa N, Almeida OFX, Van Dam AM, Rajkowska G, Swaab DF, Czéh B: Neuropathology of stress. Acta Neuropathol 2014;127:109-135.

2 Salleh MR: Life event, stress and illness. Malaysian J Med Sci 2008;15:9-18.

3 Selye H: The Stress of Life. New York, McGraw-Hill, 1956.

4 Yonas MA, Lange NE, Celedón JC: Psychosocial stress and asthma morbidity. Curr Opin Allergy Clin Immunol 2012;12:202-210.

5 Bartel DP: MicroRNAs: genomics, biogenesis, mechanism, and function. Cell 2004;116:281-297.

6 Bartel DP: MicroRNAs: target recognition and regulatory functions. Cell 2009;136:215-233.

7 Lee Y, Kim M, Han J, Yeom K-H, Lee S, Baek SH, Kim VN: MicroRNA genes are transcribed by RNA polymerase II. EMBO J 2004;23:4051-4060.

8 He L, Hannon GJ: MicroRNAs: small RNAs with a big role in gene regulation. Nat Rev Genet 2004;5:522-531.

-9 Griffiths-Jones S, Grocock RJ, van Dongen S, Bateman A, Enright AJ: miRBase: microRNA sequences, targets and gene nomenclature. Nucleic Acids Res 2006;34:D140-D144.

10 Selbach M, Schwanhäusser B, Thierfelder N, Fang Z, Khanin R, Rajewsky N: Widespread changes in protein synthesis induced by microRNAs. Nature 2008;455:58-63.

11 Tsai LM, Yu D: MicroRNAs in common diseases and potential therapeutic applications. Clin Exp Pharmacol Physiol 2010;37:102-107.

12 Dai R, Ahmed SA: MicroRNA, a new paradigm for understanding immunoregulation, inflammation, and autoimmune diseases. Transl Res 2011;157:163-179.

13 Katsuura S, Kuwano Y, Yamagishi N, Kurokawa K, Kajita K, Akaike Y, Nishida K, Masuda K, Tanahashi T, Rokutan K: MicroRNAs miR-144/144* and miR-16 in peripheral blood are potential biomarkers for naturalistic stress in healthy Japanese medical students. Neurosci Lett 2012;516:79-84.

14 Meerson A, Cacheaux L, Goosens KA, Sapolsky RM, Soreq H, Kaufer D: Changes in brain MicroRNAs contribute to cholinergic stress reactions. J Mol Neurosci 2010;40:47-55.

15 Leung AKL, Sharp PA: microRNAs: a safeguard against turmoil? Cell 2007;130:581-585.

16 Leung AKL, Sharp PA: MicroRNA functions in stress responses. Mol Cell 2010;40:205-215.

17 Dwivedi Y: Emerging role of microRNAs in major depressive disorder: diagnosis and therapeutic implications. Dialogues Clin Neurosci 2014;16:43-61.

18 Malan-Müller S, Hemmings SMJ, Seedat S: Big effects of small RNAs: a review of microRNAs in anxiety. Mol Neurobiol 2013;47:726-739.

19 Falagas ME, Vouloumanou EK, Mavros MN, Karageorgopoulos DE: Economic crises and mortality: a review of the literature. Int J Clin Pract 2009;63:1128-1135.

-20 Michael A, Bajracharya SD, Yuen PST, Zhou H, Star RA, Illei GG, Alevizos I: Exosomes from human saliva as a source of microRNA biomarkers. Oral Dis 2010;16:34-38.

21 Vaisvaser S, Modai S, Farberov L, Lin T, Sharon H, Gilam A, Volk N, Admon R, Edry L, Fruchter E, Wald I, Bar-Haim Y, Tarrasch R, Chen A, Shomron N, Hendler T: Neuro-epigenetic indications of acute stress response in humans: the case of microRNA-29c. PLoS One 2016;11:e0146236-e0146236.

-22 Beech RD, Leffert JJ, Lin A, Hong KA, Hansen J, Umlauf S, Mane S, Zhao H, Sinha R: Stress-related alcohol consumption in heavy drinkers correlates with expression of miR-10a, miR-21, and components of the TARRNA-binding protein-associated complex. Alcohol Clin Exp Res 2014;38:2743-2753.

-23 Honda M, Kuwano Y, Katsuura-Kamano S, Kamezaki Y, Fujita K, Akaike Y, Kano S, Nishida K, Masuda K, Rokutan K: Chronic academic stress increases a group of microRNAs in peripheral blood. PLoS One 2013;8:e75960e75960.

24 Gidron Y, De Zwaan M, Quint K, Ocker M: Influence of stress and health-behaviour on miRNA expression. Mol Med Rep 2010;3:455-457.

25 Hunsberger JG, Austin DR, Chen G, Manji HK: MicroRNAs in mental health: from biological underpinnings to potential therapies. Neuromol Med 2009;11:173-182.

26 Julian LJ: Measures of anxiety: State-Trait Anxiety Inventory (STAI), Beck Anxiety Inventory (BAI), and Hospital Anxiety and Depression Scale-Anxiety (HADS-A). Arthritis Care Res 2011;63(suppl 1):S467-S472.

27 Spielberger CD, Gorsuch RL, Lushene RE, Vagg PR, Jacobs GA: Manual for the State-Trait Anxiety Inventory (Form Y1-Y2). Paolo Alto, Consulting Psychologists Press, 1983.

-28 Leidinger P, Backes C, Deutscher S, Schmitt K, Mueller SC, Frese K, Haas J, Ruprecht K, Paul F, Stähler C, Lang CJG, Meder B, Bartfai T, Meese E, Keller A: A blood based 12-miRNA signature of Alzheimer disease patients. Genome Biol 2013;14:R78-R78.

29 Weigelt K, Bergink V, Burgerhout KM, Pescatori M, Wijkhuijs A, Drexhage HA: Down-regulation of inflammation-protective microRNAs $146 \mathrm{a}$ and 212 in monocytes of patients with postpartum psychosis. Brain Behav Immun 2013;29:147-155.

30 Sarachana T, Zhou R, Chen G, Manji HK, Hu VW: Investigation of post-transcriptional gene regulatory networks associated with autism spectrum disorders by microRNA expression profiling of lymphoblastoid cell lines. Genome Med 2010;2:23-23.

31 Levenstein S, Prantera C, Varvo V, Scribano ML, Berto E, Luzi C, Andreoli A: Development of the Perceived Stress Questionnaire: a new tool for psychosomatic research. J Psychosom Res 1993;37:19-32. 
32 Bertera EM: Development and validation of a short health behaviors index for use with low socioeconomic status (SES) older adults. J Clin Geropsychol 2002;8:101-115.

-33 Gu H-F, Tang C-K, Yang Y-Z: Psychological stress, immune response, and atherosclerosis. Atherosclerosis 2012;223:69-77.

34 Bibbey A, Carroll D, Roseboom TJ, Phillips AC, de Rooij SR: Personality and physiological reactions to acute psychological stress. Int J Psychophysiol 2013;90:28-36.

35 Frisch JU, Häusser JA, Mojzisch A: The Trier Social Stress Test as a paradigm to study how people respond to threat in social interactions. Front Psychol 2015;6:14.

-36 Roifman I, Beck PL, Anderson TJ, Eisenberg MJ, Genest J: Chronic inflammatory diseases and cardiovascular risk: a systematic review. Can J Cardiol 2011;27:174-182.

-37 Allen AP, Kennedy PJ, Cryan JF, Dinan TG, Clarke G: Biological and psychological markers of stress in humans: focus on the Trier Social Stress Test. Neurosci Biobehav Rev 2014;38:94-124.

38 Gordis EB, Granger DA, Susman EJ, Trickett PK: Asymmetry between salivary cortisol and alpha-amylase reactivity to stress: relation to aggressive behavior in adolescents. Psychoneuroendocrinology 2006;31:976-987.

39 Birkett MA: The Trier Social Stress Test protocol for inducing psychological stress. J Vis Exp 2011:56;3238.

-40 Hayashi T: Conversion of psychological stress into cellular stress response: roles of the sigma- 1 receptor in the process. Psychiatry Clin Neurosci 2015;69:179-191.

41 Kultz D: Evolution of the cellular stress proteome: from monophyletic origin to ubiquitous function. J Exp Biol 2003;206:3119-3124.

-42 Fulda S, Gorman AM, Hori O, Samali A: Cellular stress responses: cell survival and cell death. Int J Cell Biol 2010;2010:214074.

43 Picard M, Juster RP, McEwen BS: Mitochondrial allostatic load puts the 'gluc' back in glucocorticoids. Nat Rev Endocrinol 2014;10:303-310.

44 Simon NM, Smoller JW, McNamara KL, Maser RS, Zalta AK, Pollack MH, Nierenberg AA, Fava M, Wong KK: Telomere shortening and mood disorders: preliminary support for a chronic stress model of accelerated aging. Biol Psychiatry 2006;60:432-435.

45 Osler M, Bendix L, Rask L, Rod NH: Stressful life events and leucocyte telomere length: Do lifestyle factors, somatic and mental health, or low grade inflammation mediate this relationship? Results from a cohort of Danish men born in 1953. Brain Behav Immun 2016;58:248-253.

-46 Hanssen LM, Schutte NS, Malouff JM, Epel ES: The relationship between childhood psychosocial stressor level and telomere length: a meta-analysis. Health Psychol Res 2017;5:6378.

47 Kinoshita C, Aoyama K, Nakaki T: microRNA as a new agent for regulating neuronal glutathione synthesis and metabolism. AIMS Mol Sci 2015;1:124-143.

48 Zhang X, Ng W-L, Wang P, Tian L, Werner E, Wang H, Doetsch P, Wang Y: MicroRNA-21 modulates the levels of reactive oxygen species by targeting SOD3 and TNF $\alpha$. Cancer Res 2012;72:4707-4713.

-49 Chitnis N, Pytel D, Diehl JA: UPR-inducible miRNAs contribute to stressful situations. Trends Biochem Sci 2013;38:447-452.

50 Roufayel R, Johnston DS, Mosser DD: The elimination of miR-23a in heat-stressed cells promotes NOXAinduced cell death and is prevented by HSP70. Cell Death Dis 2014;5:e1546-e1546.

51 Chitnis NS, Pytel D, Bobrovnikova-Marjon E, Pant D, Zheng H, Maas NL, Frederick B, Kushner JA, Chodosh LA, Koumenis C, Fuchs SY, Diehl JA: miR-211 is a prosurvival microRNA that regulates chop expression in a PERKdependent manner. Mol Cell 2012;48:353-364.

52 Yan S, Sorrell M, Berman Z: Functional interplay between ATM/ATR-mediated DNA damage response and DNA repair pathways in oxidative stress. Cell Mol Life Sci 2014;71:3951-3967.

53 Issler O, Haramati S, Paul ED, Maeno H, Navon I, Zwang R, Gil S, Mayberg HS, Dunlop BW, Menke A, Awatramani R, Binder EB, Deneris ES, Lowry CA, Chen A: MicroRNA 135 is essential for chronic stress resiliency, antidepressant efficacy, and intact serotonergic activity. Neuron 2014;83:344-360.

54 Robertson S, MacKenzie SM, Alvarez-Madrazo S, Diver LA, Lin J, Stewart PM, Fraser R, Connell JM, Davies E: MicroRNA-24 is a novel regulator of aldosterone and cortisol production in the human adrenal cortex. Hypertension 2013;62:572-578.

55 Dirven BCJ, Homberg JR, Kozicz T, Henckens M: Epigenetic programming of the neuroendocrine stress response by adult life stress. J Mol Endocrinol 2017;59:R11-R31.

56 Rinaldi A, Vincenti S, De Vito F, Bozzoni I, Oliverio A, Presutti C, Fragapane P, Mele A: Stress induces region specific alterations in microRNAs expression in mice. Behav Brain Res 2010;208:265-269.

57 Krek A, Grün D, Poy MN, Wolf R, Rosenberg L, Epstein EJ, MacMenamin P, da Piedade I, Gunsalus KC, Stoffel M, Rajewsky N: Combinatorial microRNA target predictions. Nat Genet 2005;37:495-500.

58 Mannironi C, Camon J, De Vito F, Biundo A, De Stefano ME, Persiconi I, Bozzoni I, Fragapane P, Mele A, Presutti C: Acute stress alters amygdala microRNA miR-135a and miR-124 expression: inferences for corticosteroid dependent stress response. PLoS One 2013;8:e73385-e73385.

-59 Cui Y, Bai Y, Wang XD, Liu B, Zhao Z, Wang LS: Differential expression of miRNA in rat myocardial tissues under psychological and physical stress. Exp Ther Med 2014;7:901-906.

60 Balakathiresan NS, Chandran R, Bhomia M, Jia M, Li H, Maheshwari RK: Serum and amygdala microRNA signatures of posttraumatic stress: fear correlation and biomarker potential. J Psychiatr Res 2014;57:65-73.

-61 Snyder JS, Soumier A, Brewer M, Pickel J, Cameron HA: Adult hippocampal neurogenesis buffers stress responses and depressive behaviour. Nature 2011;476:458-461. 
-62 Anacker C, Cattaneo A, Musaelyan K, Zunszain PA, Horowitz M, Molteni R, Luoni A, Calabrese F, Tansey K, Gennarelli M, Thuret S, Price J, Uher R, Riva MA, Pariante CM: Role for the kinase SGK1 in stress, depression, and glucocorticoid effects on hippocampal neurogenesis. Proc Nat Acad Sci USA 2013;110:8708-8713.

63 Smalheiser NR, Lugli G, Rizavi HS, Torvik VI, Turecki G, Dwivedi Y: MicroRNA expression is down-regulated and reorganized in prefrontal cortex of depressed suicide subjects. PLoS One 2012;7:e33201-e33201.

-64 Wan Y, Liu Y, Wang X, Wu J, Liu K, Zhou J, Liu L, Zhang C: Identification of differential microRNAs in cerebrospinal fluid and serum of patients with major depressive disorder. PLoS One 2015;10:e0121975-e0121975.

65 Wei H, Yuan Y, Liu S, Wang C, Yang F, Lu Z, Wang C, Deng H, Zhao J, Shen Y, Zhang C, Yu X, Xu Q: Detection of circulating miRNA levels in schizophrenia. Am J Psychiatry 2015;172:1141-1147.

66 Mundalil Vasu M, Anitha A, Thanseem I, Suzuki K, Yamada K, Takahashi T, Wakuda T, Iwata K, Tsujii M, Sugiyama T, Mori N: Serum microRNA profiles in children with autism. Mol Autism 2014;5:40.

67 Walker RM, Rybka J, Anderson SM, Torrance HS, Boxall R, Sussmann JE, Porteous DJ, McIntosh AM, Evans KL: Preliminary investigation of miRNA expression in individuals at high familial risk of bipolar disorder. J Psychiatr Res 2015;62:48-55.

68 Kiko T, Nakagawa K, Tsuduki T, Furukawa K, Arai H, Miyazawa T: MicroRNAs in plasma and cerebrospinal fluid as potential markers for Alzheimer's disease. J Alzheimers Dis 2014;39:253-259.

-69 Sheinerman KS, Tsivinsky VG, Crawford F, Mullan MJ, Abdullah L, Umansky SR: Plasma microRNA biomarkers for detection of mild cognitive impairment. Aging 2012;4:590-605.

-70 Serafini G, Pompili M, Hansen KF, Obrietan K, Dwivedi Y, Shomron N, Girardi P: The involvement of microRNAs in major depression, suicidal behavior, and related disorders: a focus on miR-185 and miR-491-3p. Cell Mol Neurobiol 2014;34:17-30.

71 Taouis M: MicroRNAs in the hypothalamus. Best Pract Res Clin Endocrinol Metab 2016;30:641-651.

72 Shen J, Todd NW, Zhang H, Yu L, Lingxiao X, Mei Y, Guarnera M, Liao J, Chou A, Lu CL, Jiang Z, Fang H, Katz RL, Jiang F: Plasma microRNAs as potential biomarkers for non-small-cell lung cancer. Lab Invest 2011;91:579_ 587.

73 Weber JA, Baxter DH, Zhang S, Huang DY, Huang KH, Lee MJ, Galas DJ, Wang K: The microRNA spectrum in 12 body fluids. Clinical Chemistry 2010;56:1733-1741.

-74 Allegra A, Alonci A, Campo S, Penna G, Petrungaro A, Gerace D, Musolino C: Circulating microRNAs: new biomarkers in diagnosis, prognosis and treatment of cancer (review). Int J Oncol 2012;41:1897-1912.

-75 Lin Q, Mao W, Shu Y, Lin F, Liu S, Shen H, Gao W, Li S, Shen D: A cluster of specified microRNAs in peripheral blood as biomarkers for metastatic non-small-cell lung cancer by stem-loop RT-PCR. J Cancer Res Clin Oncol 2012;138:85-93.

-76 Lässer C, Eldh M, Lötvall J: Isolation and characterization of RNA-containing exosomes. J Vis Exp 2012; 59:e3037.

77 Cui SF, Li W, Niu J, Zhang CY, Chen X, Ma JZ: Acute responses of circulating microRNAs to low-volume sprint interval cycling. Front Physiol 2015;6:311.

78 Liu Y, Gao G, Yang C, Zhou K, Shen B, Liang H, Jiang X: The role of circulating microRNA-126 (miR-126): a novel biomarker for screening prediabetes and newly diagnosed type 2 diabetes mellitus. Int J Mol Sci 2014;15: 10567-10577.

79 Churov AV, Oleinik EK, Knip M: MicroRNAs in rheumatoid arthritis: altered expression and diagnostic potential. Autoimmun Rev 2015;14:1029-1037.

-80 Patel RS, Jakymiw A, Yao B, Pauley BA, Carcamo WC, Katz J, Cheng JQ, Chan EKL: High resolution of microRNA signatures in human whole saliva. Arch Oral Biol 2011;56:1506-1513.

-81 Park NJ, Zhou H, Elashoff D, Henson BS, Kastratovic DA, Abemayor E, Wong DT: Salivary microRNA: discovery, characterization, and clinical utility for oral cancer detection. Clin Cancer Res 2009;15:5473-5477.

-82 Xie Z, Chen G, Zhang X, Li D, Huang J, Yang C, Zhang P, Qin Y, Duan Y, Gong B, Li Z: Salivary microRNAs as promising biomarkers for detection of esophageal cancer. PLoS One 2013;8:e57502-e57502.

-83 Xie Z, Yin X, Gong B, Nie W, Wu B, Zhang X, Huang J, Zhang P, Zhou Z, Li Z: Salivary microRNAs show potential as a noninvasive biomarker for detecting resectable pancreatic cancer. Cancer Prev Res (Philadelphia) 2015; 8:165-173.

-84 Xie Y, Todd NW, Liu Z, Zhan M, Fang H, Peng H, Alattar M, Deepak J, Stass SA, Jiang F: Altered miRNA expression in sputum for diagnosis of non-small cell lung cancer. Lung Cancer 2010;67:170-176.

85 Alevizos I, Illei GG: MicroRNAs as biomarkers in rheumatic diseases. Nat Rev Rheumatol 2010;6:391-398. 